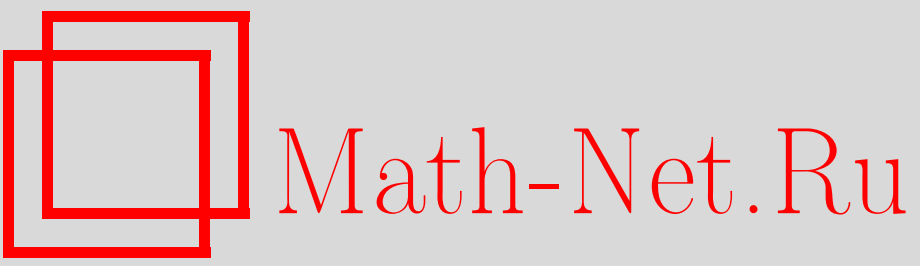

В. С. Дрюма, Приложения римановой геометрии и геометрии Эйнштейна-Вейля в теории обыкновенных дифференциальных уравнений второго порядка, ТМФ, 2001, том 128, номер 1, 15-26

DOI: https://doi.org/10.4213/tmf479

Использование Общероссийского математического портала Math-Net.Ru подразумевает, что вы прочитали и согласны с пользовательским соглашением

http: //www . mathnet.ru/rus/agreement

Параметры загрузки:

IP : 54.224 .135 .184

26 апреля 2023 г., 14:18:49 


\section{ПРИЛОЖЕНИЯ РИМАНОВОЙ ГЕОМЕТРИИ И ГЕОМЕТРИИ ЭЙНШТЕЙНА-ВЕЙЛЯ В ТЕОРИИ ОБЫКНОВЕННЫХ ДИФФЕРЕНЦИАЛЬНЫХ УРАВНЕНИЙ ВТОРОГО ПОРЯДКА}

Рассмотрены некоторые свойства четырехмерных римановых пространств, метрические коэффициенты которых связаны с коэффициентами нелинейных дифференциальных уравнений второго порядка. Исследованы свойства трехмерных пространств Эйнштейна-Вейля, связанных с дуальными уравнениями $b^{\prime \prime}=g\left(a, b, b^{\prime}\right)$, в которых функция $g\left(a, b, b^{\prime}\right)$ удовлетворяет специальному дифференциальному уравнению в частных производных.

\section{1. ВВЕДЕНИЕ}

Обыкновенные дифференциальные уравнения (ОДУ) второго порядка и вида

$$
y^{\prime \prime}+a_{1}(x, y) y^{\prime 3}+3 a_{2}(x, y) y^{\prime 2}+3 a_{3}(x, y) y^{\prime}+a_{4}(x, y)=0
$$

связаны с нелинейной динамической системой, задаваемой следуюшими формулами:

$$
\frac{d x}{d t}=P\left(x, y, z, \alpha_{i}\right), \quad \frac{d y}{d t}=Q\left(x, y, z, \alpha_{i}\right), \quad \frac{d z}{d t}=R\left(x, y, z, \alpha_{i}\right),
$$

где $\alpha_{i}$ - некоторые параметры. Например, система Лоренца

$$
\dot{X}=\sigma(Y-X), \quad \dot{Y}=r X-Y-Z X, \quad \dot{Z}=X Y-b Z,
$$

проявляющая свойства хаотической системы при некоторых значениях параметров $\sigma$, $r, b$, оказывается эквивалентной уравнению

$$
y^{\prime \prime}-\frac{3}{y} y^{\prime 2}+\left(\alpha y-\frac{1}{x}\right) y^{\prime}+\epsilon x y^{4}-\beta x^{3} y^{4}-\beta x^{2} y^{3}-\gamma y^{3}+\delta \frac{y^{2}}{x}=0,
$$

где

$$
\alpha=\frac{b+\sigma+1}{\sigma}, \quad \beta=\frac{1}{\sigma^{2}}, \quad \gamma=\frac{b(\sigma+1)}{\sigma^{2}}, \quad \delta=\frac{(\sigma+1)}{\sigma}, \quad \epsilon=\frac{b(r-1)}{\sigma^{2}} .
$$

\footnotetext{
${ }^{*}$ Институт математики и информатики, Кишинев, Молдова. E-mail: valery@gala.moldova.su
} 
Свойства этого уравнения были исследованы в работах [1]-[5] с использованием теории инвариантов. В соответствии с этой теорией [6]-[10] все уравнения вида (1) могут быть разбиты на два класса в зависимости от того, равен или не равен нулю коэффициент $\nu_{5}$ :

$$
\nu_{5}=L_{2}\left(L_{1} L_{2 x}-L_{2} L_{1 x}\right)+L_{1}\left(L_{2} L_{1 y}-L_{1} L_{2 y}\right)-a_{1} L_{1}^{3}+3 a_{2} L_{1}^{2} L_{2}-3 a_{3} L_{1} L_{2}^{2}+a_{4} L_{2}^{3},
$$

где $L_{1}$ и $L_{2}$, в свою очередь, задаются формулами

$$
\begin{aligned}
& L_{1}=\frac{\partial}{\partial y}\left(a_{4 y}+3 a_{2} a_{4}\right)-\frac{\partial}{\partial x}\left(2 a_{3 y}-a_{2 x}+a_{1} a_{4}\right)-3 a_{3}\left(2 a_{3 y}-a_{2 x}\right)-a_{4} a_{1 x} \\
& L_{2}=\frac{\partial}{\partial x}\left(a_{1 x}-3 a_{1} a_{3}\right)+\frac{\partial}{\partial x}\left(a_{3 y}-2 a_{2 x}+a_{1} a_{4}\right)-3 a_{2}\left(a_{3 y}-2 a_{2 x}\right)+a_{1} a_{4 y} .
\end{aligned}
$$

Для уравнений с $\nu_{5}=0$ Лиувилль открыл набор полуинвариантов, начинающихся с

$$
w_{1}=\frac{1}{L_{1}^{4}}\left[L_{1}^{3}\left(\alpha^{\prime} L_{1}-\alpha^{\prime \prime} L_{2}\right)+R_{1}\left(L_{1}^{2}\right)_{x}-L_{1}^{2} R_{1 x}+L_{1} R_{1}\left(a_{3} L_{1}-a_{4} L_{2}\right)\right],
$$

где $R_{1}=L_{1} L_{2 x}-L_{2} L_{1 x}+a_{2} L_{1}^{2}-2 a_{3} L_{1} L_{2}+a_{4} L_{2}^{2}$ и

$$
\begin{aligned}
\alpha & =a_{2 y}-a_{1 x}+2\left(a_{1} a_{3}-a_{2}^{2}\right), \\
\alpha^{\prime} & =a_{3 y}-a_{2 x}+a_{1} a_{4}-a_{2} a_{3}, \\
\alpha^{\prime \prime} & =a_{4 y}-a_{3 x}+2\left(a_{2} a_{4}-a_{3}^{2}\right) .
\end{aligned}
$$

В случае, когда $\nu_{5} \neq 0$, полуинварианты имеют вид

$$
\nu_{m+5}=L_{1} \frac{\partial \nu_{m}}{\partial y}-L_{2} \frac{\partial \nu_{m}}{\partial x}+m \nu_{m}\left(\frac{\partial L_{2}}{\partial x}-\frac{\partial L_{1}}{\partial y}\right)
$$

а соответствуюшая серия полных инвариантов задается формулой

$$
\left[5 t_{m}-(m-2) t_{7} t_{m-2}\right] \nu_{5}^{2 / 5}=5\left(L_{1} \frac{\partial t_{m-2}}{\partial y}-L_{2} \frac{\partial t_{m-2}}{\partial x}\right)
$$

где $t_{m}=\nu_{m} \nu_{5}^{-m / 5}$.

\section{2. РИМАНОВЫ ПРОСТРАНСТВА И ТЕОРИЯ ОДУ}

Для построения римановых пространств, связанных с уравнением (1), выпишем уравнения геодезических в двумерном пространстве $A_{2}$, снабженном аффинной (или римановой) связностью,

$$
\begin{aligned}
& \ddot{x}+\Gamma_{11}^{1} \dot{x}^{2}+2 \Gamma_{12}^{1} \dot{x} \dot{y}+\Gamma_{22}^{1} \dot{y}^{2}=0, \\
& \ddot{y}+\Gamma_{11}^{2} \dot{x}^{2}+2 \Gamma_{12}^{2} \dot{x} \dot{y}+\Gamma_{22}^{2} \dot{y}^{2}=0 .
\end{aligned}
$$

Эта система уравнений эквивалентна единственному уравнению

$$
y^{\prime \prime}-\Gamma_{22}^{1} y^{\prime 3}+\left(\Gamma_{22}^{2}-2 \Gamma_{12}^{1}\right) y^{\prime 2}+\left(2 \Gamma_{12}^{2}-\Gamma_{11}^{1}\right) y^{\prime}+\Gamma_{11}^{2}=0
$$


которое является уравнением типа (1) со специальным выбором коэффициентов $a_{i}(x, y)$.

Уравнение (1) с произвольными коэффициентами $a_{i}(x, y)$ может быть интерпретировано как уравнение геодезической в двумерном пространстве $A_{2}$ :

$$
\begin{aligned}
& \ddot{x}-a_{3} \dot{x}^{2}-2 a_{2} \dot{x} \dot{y}-a_{1} \dot{y}^{2}=0, \\
& \ddot{y}+a_{4} \dot{x}^{2}+2 a_{3} \dot{x} \dot{y}+a_{2} \dot{y}^{2}=0,
\end{aligned}
$$

проективная связность которого имеет компоненты

$$
\Pi_{1}=\left|\begin{array}{cc}
-a_{3} & -a_{2} \\
a_{4} & a_{3}
\end{array}\right|, \quad \Pi_{2}=\left|\begin{array}{cc}
-a_{2} & -a_{1} \\
a_{3} & a_{2}
\end{array}\right| .
$$

Тензор кривизны связности такого типа имеет вид

$$
R_{12}=\frac{\partial \Pi_{2}}{\partial x}-\frac{\partial \Pi_{1}}{\partial y}+\left[\Pi_{1}, \Pi_{2}\right]
$$

а его компоненты выражаются формулами

$$
\begin{array}{ll}
R_{112}^{1}=a_{3 y}-a_{2 x}+a_{1} a_{4}-a_{2} a_{3}=\alpha^{\prime}, & R_{212}^{1}=a_{2 y}-a_{1 x}+2\left(a_{1} a_{3}-a_{2}^{2}\right)=\alpha, \\
R_{112}^{2}=a_{3 x}-a_{4 y}+2\left(a_{3}^{2}-a_{2} a_{4}\right)=-\alpha^{\prime \prime}, & R_{212}^{2}=a_{2 x}-a_{3 y}+a_{3} a_{2}-a_{1} a_{4}=-\alpha^{\prime} .
\end{array}
$$

Для построения риманова пространства, связанного с уравнением вида (1), используется риманово расширение $W^{4}$ пространства $A_{2}$ со связностью $\Pi_{i j}^{k}$ [11]. Соответствующая метрика имеет вид

$$
d s^{2}=-2 \Pi_{i j}^{k} \xi_{k} d x^{i} d x^{j}+2 d \xi_{i} d x^{i}
$$

В исследуемом случае (когда $\xi_{1}=z$ и $\xi_{2}=\tau$ ) эта метрика дается формулой

$$
d s^{2}=2\left(z a_{3}-\tau a_{4}\right) d x^{2}+4\left(z a_{2}-\tau a_{3}\right) d x d y+2\left(z a_{1}-\tau a_{2}\right) d y^{2}+2 d x d z+2 d y d \tau
$$

Теперь можно сформулировать утверждение.

ПРЕДЛОЖЕНИЕ 1. Для заданного уравнения типа (1) получим риманово пространство с метрикой (3), часть геодезических кривых которого суть интегральнье кривые данного уравнения. 
Вычисляя геодезические для пространства $W^{4}$ с метрикой (3), получим следующую систему уравнений:

$$
\begin{aligned}
& \frac{d^{2} x}{d s^{2}}-a_{3}\left(\frac{d x}{d s}\right)^{2}-2 a_{2} \frac{d x}{d s} \frac{d y}{d s}-a_{1}\left(\frac{d y}{d s}\right)^{2}=0 \\
& \frac{d^{2} y}{d s^{2}}+a_{4}\left(\frac{d x}{d s}\right)^{2}+2 a_{3} \frac{d x}{d s} \frac{d y}{d s}+a_{2}\left(\frac{d y}{d s}\right)^{2}=0 \\
& \frac{d^{2} z}{d s^{2}}+\left[z\left(a_{4 y}-\alpha^{\prime \prime}\right)-\tau a_{4 x}\right]\left(\frac{d x}{d s}\right)^{2}+ \\
&+2\left[z a_{3 y}-\tau\left(a_{3 x}+\alpha^{\prime \prime}\right)\right] \frac{d x}{d s} \frac{d y}{d s}+\left[z\left(a_{2 y}+\alpha\right)-\tau\left(a_{2 x}+2 \alpha^{\prime}\right)\right]\left(\frac{d y}{d s}\right)^{2}+ \\
&+2 a_{3} \frac{d x}{d s} \frac{d z}{d s}-2 a_{4} \frac{d x}{d s} \frac{d \tau}{d s}+2 a_{2} \frac{d y}{d s} \frac{d z}{d s}-2 a_{3} \frac{d y}{d s} \frac{d \tau}{d s}=0, \\
& \frac{d^{2} \tau}{d s^{2}+}\left[z\left(a_{3 y}-2 \alpha^{\prime}\right)-\tau\left(a_{3 x}-\alpha^{\prime \prime}\right)\right]\left(\frac{d x}{d s}\right)^{2}+ \\
&+2\left[z\left(a_{2 y}-\alpha\right)-\tau a_{2 x}\right] \frac{d x}{d s} \frac{d y}{d s}+\left[z a_{1 y}-\tau\left(a_{1 x}+\alpha\right)\right]\left(\frac{d y}{d s}\right)^{2}+ \\
&+2 a_{2} \frac{d x}{d s} \frac{d z}{d s}-2 a_{3} \frac{d x}{d s} \frac{d \tau}{d s}+2 a_{1} \frac{d y}{d s} \frac{d z}{d s}-2 a_{2} \frac{d y}{d s} \frac{d \tau}{d s}=0 .
\end{aligned}
$$

Эта система уравнений имеет интеграл движения

$$
2\left(z a_{3}-\tau a_{4}\right) \dot{x}^{2}+4\left(z a_{2}-\tau a_{3}\right) \dot{x} \dot{y}+2\left(z a_{1}-\tau a_{2}\right) \dot{y}^{2}+2 \dot{x} \dot{z}+2 \dot{y} \dot{\tau}=1 .
$$

Заметим также, что первые два уравнения этой системы эквивалентны уравнению (1).

Таким образом, построено четырехмерное риманово пространство с метрикой (3) и связностью

$$
\begin{aligned}
& \Gamma_{1}=\left|\begin{array}{cccc}
-a_{3} & -a_{2} & 0 & 0 \\
a_{4} & a_{3} & 0 & 0 \\
z\left(a_{4 y}-\alpha^{\prime \prime}\right)-\tau a_{4 x} & z a_{3 y}-\tau\left(a_{3 x}+\alpha^{\prime \prime}\right) & a_{3} & -a_{4} \\
z\left(a_{3 y}-2 \alpha^{\prime}\right)-\tau\left(a_{3 x}-\alpha^{\prime \prime}\right) & z\left(a_{2 y}-\alpha\right)-\tau a_{2 x} & a_{2} & -a_{3}
\end{array}\right| \\
& \Gamma_{2}=\left|\begin{array}{cccc}
-a_{2} & -a_{1} & 0 & 0 \\
a_{3} & a_{2} & 0 & 0 \\
z a_{3 y}-\tau\left(a_{3 x}+\alpha^{\prime \prime}\right) & z\left(a_{2 y}+\alpha\right)-\tau\left(a_{2 x}+2 \alpha^{\prime}\right) & a_{2} & -a_{3} \\
z\left(a_{2 y}-\alpha\right)-\tau a_{2 x} & z a_{1 y}-\tau\left(a_{1 x}+\alpha\right) & a_{1} & -a_{2}
\end{array}\right|, \\
& \Gamma_{3}=\left|\begin{array}{cccc}
0 & 0 & 0 & 0 \\
0 & 0 & 0 & 0 \\
a_{3} & a_{2} & 0 & 0 \\
a_{2} & a_{1} & 0 & 0
\end{array}\right|, \quad \Gamma_{4}=\left|\begin{array}{cccc}
0 & 0 & 0 & 0 \\
0 & 0 & 0 & 0 \\
-a_{4} & -a_{3} & 0 & 0 \\
-a_{3} & -a_{2} & 0 & 0
\end{array}\right|
\end{aligned}
$$


Тензор кривизны этой метрики имеет вид

$$
\begin{aligned}
& R_{112}^{1}=-R_{312}^{3}=-R_{212}^{2}=R_{412}^{4}=\alpha^{\prime}, \\
& R_{212}^{1}=-R_{312}^{4}=\alpha, \quad R_{112}^{2}=-R_{412}^{3}=-\alpha^{\prime \prime}, \\
& R_{312}^{1}=R_{412}^{1}=R_{312}^{2}=R_{412}^{2}=0, \\
& R_{112}^{3}=2 z\left(a_{2} \alpha^{\prime \prime}-a_{3} \alpha^{\prime}\right)+2 \tau\left(a_{4} \alpha^{\prime}-a_{3} \alpha^{\prime \prime}\right), \\
& R_{212}^{4}=2 z\left(a_{3} \alpha^{\prime}-a_{2} \alpha\right)+2 \tau\left(a_{3} \alpha-a_{2} \alpha^{\prime}\right), \\
& R_{212}^{3}=z\left(\alpha_{x}-\alpha_{y}^{\prime}+a_{1} \alpha^{\prime \prime}-a_{3} \alpha\right)+\tau\left(\alpha_{y}^{\prime \prime}-\alpha_{x}^{\prime}+a_{4} \alpha-a_{2} \alpha^{\prime \prime}\right), \\
& R_{112}^{4}=z\left(\alpha_{y}^{\prime}-\alpha_{x}+a_{1} \alpha^{\prime \prime}-a_{3} \alpha\right)+\tau\left(\alpha_{x}^{\prime}-\alpha_{y}^{\prime \prime}+a_{4} \alpha-a_{2} \alpha^{\prime \prime}\right) .
\end{aligned}
$$

Используя следующие выражения для компонент проективной кривизны пространства $A_{2}$ :

$$
\begin{aligned}
& L_{1}=\alpha_{y}^{\prime \prime}-\alpha_{x}^{\prime}+a_{2} \alpha^{\prime \prime}+a_{4} \alpha-2 a_{3} \alpha^{\prime}, \\
& L_{2}=\alpha_{y}^{\prime}-\alpha_{x}+a_{1} \alpha^{\prime \prime}+a_{3} \alpha-2 a_{2} \alpha^{\prime},
\end{aligned}
$$

можно записать компоненты тензора кривизны:

$$
\begin{aligned}
R_{112}^{4} & =z\left(L_{2}+2 a_{2} \alpha^{\prime}-2 a_{3} \alpha\right)-\tau\left(L_{1}+2 a_{3} \alpha^{\prime}-2 a_{4} \alpha\right), \\
R_{212}^{3} & =z\left(-L_{2}+2 a_{1} \alpha^{\prime \prime}-2 a_{2} \alpha^{\prime}\right)+\tau\left(L_{1}+2 a_{3} \alpha^{\prime}-2 a_{2} \alpha^{\prime \prime}\right), \\
R_{13} & =\left|\begin{array}{cccc}
0 & 0 & 0 & 0 \\
0 & 0 & 0 & 0 \\
0 & -\alpha^{\prime} & 0 & 0 \\
\alpha^{\prime} & 0 & 0 & 0
\end{array}\right|, \quad R_{14}=\left|\begin{array}{cccc}
0 & 0 & 0 & 0 \\
0 & 0 & 0 & 0 \\
0 & \alpha^{\prime \prime} & 0 & 0 \\
-\alpha^{\prime \prime} & 0 & 0 & 0
\end{array}\right|, \\
R_{23} & =\left|\begin{array}{cccc}
0 & 0 & 0 & 0 \\
0 & 0 & 0 & 0 \\
0 & -\alpha & 0 & 0 \\
\alpha & 0 & 0 & 0
\end{array}\right|, \quad R_{24}=\left|\begin{array}{cccc}
0 & 0 & 0 & 0 \\
0 & 0 & 0 & 0 \\
0 & \alpha^{\prime} & 0 & 0 \\
-\alpha^{\prime} & 0 & 0 & 0
\end{array}\right|, \\
R_{j 34}^{i} & =0 .
\end{aligned}
$$

Компоненты тензора Риччи $R_{i k}=R_{i l k}^{l}$ пространства $M^{4}$ имеют вид

$$
R_{11}=2 \alpha^{\prime \prime}, \quad R_{12}=2 \alpha^{\prime}, \quad R_{22}=2 \alpha,
$$

а скалярная кривизна $R=g^{i n} g^{k m} R_{n m}$ пространства $M^{4}$ обрашается в нуль: $R=0$.

Введем теперь тензор

$$
L_{i j k}=\nabla_{k} R_{i j}-\nabla_{j} R_{i k}=R_{i j ; k}-R_{i k ; j}
$$

с компонентами

$$
L_{112}=-L_{121}=2 L_{1}, \quad L_{221}=L_{212}=-2 L_{2} .
$$

Инварианты уравнения (1) могут быть выражены через ковариантные производные тензора кривизны и величины $L_{1}$ и $L_{2}$. 
Тензор Вейля пространства $M^{4}$

$$
C_{l i j k}=R_{l i j k}+\frac{1}{2}\left(g_{j l} R_{i k}+g_{i k} R_{j l}-g_{j k} R_{i l}-g_{i l} R_{j k}\right)+\frac{R}{6}\left(g_{j k} g_{i l}-g_{j l} g_{i k}\right)
$$

имеет единственную ненулевую компоненту $C_{1212}=t L_{1}-z L_{2}$. Исследуя уравнение

$$
\left|R_{a b}-\lambda g_{a b}\right|=0
$$

где матрица $R_{a b}$ строится из компонент тензора Римана

$$
\begin{gathered}
R_{1412}=\alpha^{\prime \prime}, \quad R_{2412}=\alpha^{\prime}, \quad R_{2312}=-\alpha, \quad R_{3112}=\alpha^{\prime}, \\
R_{1212}=z\left(\alpha_{x}-\alpha_{y}^{\prime}+a_{1} \alpha^{\prime \prime}-2 a_{2} \alpha^{\prime}+a_{3} \alpha\right)+t\left(\alpha_{y}^{\prime \prime}-\alpha_{x}^{\prime}-a_{4} \alpha+2 a_{3} \alpha^{\prime}-a_{3} \alpha^{\prime \prime}\right),
\end{gathered}
$$

получим, что пространство $M^{4}$ имеет тип $N$ и все инварианты второго порядка пространства $M^{4}$ обрашаются в нуль.

ЗАМЕчАниЕ 1. Пространства с метрикой (3) оказываются плоскими для уравнения (1) при условиях $\alpha=0, \alpha^{\prime}=0$ и $\alpha^{\prime \prime}=0$, наложенных на коэффициенты $a_{i}(x, y)$. Компоненты проективной кривизны для этих уравнений обрашаются в нуль $\left(L_{1}=0\right.$ и $\left.L_{2}=0\right)$, и тогда после локального преобразования эти условия принимают форму $y^{\prime \prime}=0$.

С другой стороны, имеются примеры уравнения (1) с условиями $L_{1}=0$ и $L_{2}=0$, но с функциями $\alpha \neq 0, \alpha^{\prime} \neq 0$ и $\alpha^{\prime \prime} \neq 0$. Для таких уравнений кривизны соответствуюших римановых пространств в нуль не обрашаются. В самом деле, рассматривая уравнение

$$
y^{\prime \prime}+2 e^{\varphi} y^{\prime 3}-\varphi_{y} y^{\prime 2}+\varphi_{x} y^{\prime}-2 e^{\varphi}=0,
$$

в котором функция $\varphi(x, y)$ есть решение нелинейного уравнения Вильчинского-Цищейки

$$
\varphi_{x y}=4 e^{2 \varphi}-e^{-\varphi}
$$

интегрируемого с помошью метода обратной задачи, получим, что для этого уравнения $L_{1}=0$ и $L_{2}=0$, но $\alpha \neq 0, \alpha^{\prime} \neq 0$ и $\alpha^{\prime \prime} \neq 0$.

ЗАмЕчАниЕ 2. Особенно интересно исследовать свойства пространств с метрикой (3) и уравнение (2) при коэффициентах, приводяших к хаотическому поведению решений $(\sigma=10, b=8 / 3$ и $r>24)$. В частности, интересно исследовать уравнения геодезического отклонения

$$
\frac{d^{2} \eta^{i}}{d s^{2}}+2 \Gamma_{l m}^{i} \frac{d x^{m}}{d s} \frac{d \eta^{l}}{d s}+\frac{\partial \Gamma_{k l}^{i}}{\partial x^{j}} \frac{d x^{k}}{d s} \frac{d x^{l}}{d s} \eta^{j}=0 .
$$

Рассмотрим теперь некоторые приложения теории солитонов к исследованию свойств уравнений типа (1). Эти приложения основаны на представлении метрики (3) в виде

$d s^{2}=2 z\left(a_{3} d x^{2}+2 a_{2} d x d y+a_{1} d y^{2}\right)-2 \tau\left(a_{4} d x^{2}+2 a_{3} d x d y+a_{2} d y^{2}\right)+2 d x d z+2 d y d \tau$ 
или

$$
d s^{2}=2 z d s_{1}^{2}-2 \tau d s_{2}^{2}+2 d x d z+2 d y d \tau
$$

Например, метрика, соответствуюшая уравнению

$$
y^{\prime \prime}+H^{2}(x, y) y^{\prime 3}+3 y^{\prime}=0
$$

представляется в виде

$$
d s^{2}=2 z\left(d x^{2}+H^{2} d y^{2}\right)-4 \tau d x d y+2 d x d z+2 d y d \tau
$$

содержащем двумерную часть

$$
d s_{1}^{2}=d x^{2}+H^{2} d y^{2}
$$

связанную с уравнением Кд $\Phi$

$$
K_{y}+K K_{x}+K_{x x x}=0
$$

которому удовлетворяет кривизна $K(x, y)$ метрики (4).

Метрика, отвечающая уравнению

$$
y^{\prime \prime}+y^{\prime 3}+3 \cos H(x, y) y^{\prime 2}+y^{\prime}=0,
$$

связана с интегрируемым уравнением

$$
H_{x y}=\sin H
$$

\section{3. СВЯЗЬ С ТЕОРИЕЙ ПОВЕРХНОСТЕЙ}

Метрика, отвечаюшая уравнению (1), может быть использована также для построения поверхностей. Один из возможных способов это сделать - вложить двумерные поверхности, являющиеся обобщениями поверхностей трансляции, в данное четырехмерное пространство. Уравнения на координаты $Z^{i}(x, y)$ таких поверхностей имеют вид

$$
\frac{\partial^{2} Z^{i}}{\partial x \partial y}+\Gamma_{j k}^{i} \frac{\partial Z^{j}}{\partial x} \frac{\partial Z^{k}}{\partial y}=0
$$

Тогда коэффициенты $a_{i}(x, y)$ и соответствующие ОДУ второго порядка получаются из условия совместности для этой системы.

Другая возможность исследовать двумерные поверхности в пространстве с метрикой (3) состоит в исследовании сечений

$$
x=x, \quad y=y, \quad z=z(x, y), \quad \tau=\tau(x, y)
$$

в таких пространствах. Из выражений

$$
d z=z_{x} d x+z_{y} d y, \quad d \tau=\tau_{x} d x+\tau_{y} d y
$$


можно получить метрику

$$
d s^{2}=2\left(z_{x}+z a_{3}-\tau a_{4}\right) d x^{2}+2\left(\tau_{x}+z_{y}+2 z a_{2}-2 \tau a_{3}\right) d x d y+2\left(\tau_{y}+z a_{1}-\tau a_{2}\right) d y^{2} .
$$

Ниже подобные представления используются для исследования частных случаев уравнения (1).

1. Функции $z$ и $\tau$, выбранные в виде

$$
z_{x}+z a_{3}-\tau a_{4}=0, \quad \tau_{x}+z_{y}+2 z a_{2}-2 \tau a_{3}=0, \quad \tau_{y}+z a_{1}-\tau a_{2}=0,
$$

задают плоские поверхности. С помощью подстановки $z=\Phi_{x}, \tau=\Phi_{y}$ эта система уравнений может быть приведена к системе

$$
\Phi_{x x}=a_{4} \Phi_{y}-a_{3} \Phi_{x}, \quad \Phi_{x y}=a_{3} \Phi_{y}-a_{2} \Phi_{x}, \quad \Phi_{y y}=a_{2} \Phi_{y}-a_{1} \Phi_{x},
$$

которая совместна с условиями $\alpha=0, \alpha^{\prime}=0$ и $\alpha^{\prime \prime}=0$.

2 . Выберем теперь функции $z=\Phi_{x}$ и $\tau=\Phi_{y}$, которые удовлетворяют системе уравнений

$$
\Phi_{x x}=a_{4} \Phi_{y}-a_{3} \Phi_{x}, \quad \Phi_{y y}=a_{2} \Phi_{y}-a_{1} \Phi_{x},
$$

где коэффициенты $a_{i}(x, y)$ представляются в виде

$$
a_{4}=R_{x x x}, \quad a_{3}=-R_{x y y}, \quad a_{2}=R_{x y y}, \quad a_{1}=R_{y y y} .
$$

Функция $R(x, y)$ есть решение уравнения Виттена-Дойграффа-Верлинде-Верлинде (ВДВВ)

$$
R_{x x x} R_{y y y}-R_{x x y} R_{x y y}=1
$$

Эти функции отвечают уравнению (1), записанному в виде

$$
y^{\prime \prime}-R_{y y y} y^{\prime 3}+3 R_{x y y} y^{\prime 2}-3 R_{x x y} y^{\prime}+R_{x x x}=0 .
$$

Выбирая коэффициенты $a_{i}$ в виде

$$
a_{4}=-2 \omega, \quad a_{1}=2 \omega, \quad a_{3}=\frac{\omega_{x}}{\omega}, \quad a_{2}=-\frac{\omega_{y}}{\omega}
$$

получим систему уравнений

$$
\Phi_{x x}+\frac{\omega_{x}}{\omega} \Phi_{x}+2 \omega \Phi_{y}=0, \quad \Phi_{y y}+2 \omega \Phi_{x}+\frac{\omega_{y}}{\omega} \Phi_{y}=0
$$

условие совместности которой

$$
\frac{\partial^{2} \ln \omega}{\partial x \partial y}=4 \omega^{2}+\frac{\kappa}{\omega}
$$

есть не что иное, как уравнение Вильчинского-Цицейки. 
ЗАМЕЧАНИЕ 3. Линейная система уравнений для уравнения ВДВВ задает некоторые поверхности в трехмерном проективном пространстве. Каноническая форма этой системы уравнений имеет вид [12]

$$
\begin{gathered}
\Phi_{x x}-R_{x x x} \Phi_{y}+\left(\frac{R_{x x x y}}{2}-\frac{R_{x x y}^{2}}{4}-\frac{R_{x x x} R_{x x y}}{2}\right) \Phi=0, \\
\Phi_{y y}-R_{y y y} \Phi_{x}+\left(\frac{R_{y y y x}}{2}-\frac{R_{x y y}^{2}}{4}-\frac{R_{y y y} R_{x x y}}{2}\right) \Phi=0 .
\end{gathered}
$$

Соотношения между инвариантами Вильчинского для линейной системы порождают различные типы поверхностей. Некоторые из этих инвариантов связаны с решениями уравнения ВДВВ.

ЗАмечаниЕ 4 . Рассмотрим систему уравнений $\xi_{i, j}+\xi_{j, i}=2 \Gamma_{i j}^{k} \xi_{k}$ на векторы Киллинга метрики (3). Эта система, записанная в компонентах, имеет вид

$$
\begin{gathered}
\xi_{1 x}=-a_{3} \xi_{1}+a_{4} \xi_{2}+\left(z A-t a_{4 x}\right) \xi_{3}+(z E+t F) \xi_{4}, \\
\xi_{2 y}=-a_{1} \xi_{1}+a_{2} \xi_{2}+(z C+t D) \xi_{3}+\left(z a_{1 y}-t H\right) \xi_{4}, \\
\xi_{1 y}+\xi_{2 x}=2\left[-a_{2} \xi_{1}+a_{3} \xi_{2}+\left(z a_{3 y}-t B\right) \xi_{3}+\left(z G-t a_{2 x}\right) \xi_{4}\right], \\
\xi_{1 z}+\xi_{3 x}=2\left[a_{3} \xi_{3}+a_{2} \xi_{4}\right], \quad \xi_{1 t}+\xi_{4 x}=2\left[-a_{4} \xi_{3}-a_{3} \xi_{4}\right], \\
\xi_{2 z}+\xi_{3 y}=2\left[a_{2} \xi_{3}+a_{1} \xi_{4}\right], \quad \xi_{2 t}+\xi_{4 y}=-2\left[a_{3} \xi_{3}-a_{2} \xi_{4}\right], \\
\xi_{3 z}=0, \quad \xi_{4 t}=0 .
\end{gathered}
$$

$\mathrm{B}$ частном случае, когда $\xi_{3}=\xi_{4}=0$ и $\xi_{i}=\xi_{i}(x, y)$, получается система уравнений

$$
\begin{gathered}
\xi_{1 x}=-a_{3} \xi_{1}+a_{4} \xi_{2}, \quad \xi_{2 y}=-a_{1} \xi_{1}+a_{2} \xi_{2}, \\
\xi_{1 y}+\xi_{2 x}=2\left[-a_{2} \xi_{1}+a_{3} \xi_{2}\right]
\end{gathered}
$$

которая оказывается эквивалентной системе уравнений на $z=z(x, y)$ и $\tau=\tau(x, y)$.

ЗАмечАниЕ 5. Оператор Бельтрами-Лапласа

$$
\Delta=g^{i j}\left(\frac{\partial^{2}}{\partial x^{i} \partial x^{j}}-\Gamma_{i j}^{k} \frac{\partial}{\partial x^{k}}\right)
$$

оказывается полезным при исследовании свойств метрики (3). Например, уравнение $\Delta \Psi=0$ принимает вид

$$
\left(t a_{4}-z a_{3}\right) \Psi_{z z}+2\left(t a_{3}-z a_{2}\right) \Psi_{z t}+\left(t a_{2}-z a_{1}\right) \Psi_{t t}+\Psi_{x z}+\Psi_{y t}=0,
$$

и его решения оказываются связанньми с геометрией метрики (3). Тогда, если подставить выражение $\Psi=\exp [z A+t B]$ в уравнение $\Delta \Psi=0$, получим условия $A=\Phi_{y}$, $B=-\Phi_{x}$ и

$$
a_{4} \Phi_{y}^{2}-2 a_{3} \Phi_{x} \Phi_{y}+a_{2} \Phi_{x}^{2}-\Phi_{y} \Phi_{x x}+\Phi_{x} \Phi_{x y}=0 .
$$


Используя решения уравнения эйконала

$$
g^{i j} \frac{\partial F}{\partial x^{i}} \frac{\partial F}{\partial x^{j}}=0
$$

или

$$
F_{x} F_{z}+F_{y} F_{t}-\left(t a_{4}-z a_{3}\right) F_{z} F_{z}-2\left(t a_{3}-z a_{2}\right) F_{z} F_{t}-\left(t a_{2}-z a_{1}\right) F_{t} F_{t}=0,
$$

можно исследовать свойства изотропных поверхностей в пространстве с метрикой (3). Решение этого уравнения, представленное в виде $F=A(x, y) z^{2}+B(x, y) t^{2}$, отвечает следуюшему выбору коэффициентов в уравнении (1):

$$
a_{1}=\frac{A B_{x}}{2 B^{2}}+\frac{A_{x}}{B}, \quad a_{4}=-\frac{B A_{y}}{2 A^{2}}-\frac{B_{y}}{A}, \quad a_{2}=-\frac{B_{y}}{2 B}, \quad a_{3}=\frac{A_{x}}{2 A} .
$$

ЗАмЕчАниЕ 6. Метрика (3) допускает представление в тетрадном виде $g_{i j}=$ $\omega_{i}^{a} \omega_{j}^{b} \eta_{a b}$, где

$$
\eta_{a b}=\left|\begin{array}{cccc}
0 & 0 & 1 & 0 \\
0 & 0 & 0 & 1 \\
1 & 0 & 0 & 0 \\
0 & 1 & 0 & 0
\end{array}\right|
$$

Тогда получим $d s^{2}=2 \omega^{1} \omega^{3}+2 \omega^{2} \omega^{4}$, где

$$
\begin{gathered}
\omega^{1}=d x+d y, \quad \omega^{2}=d x+d y+\frac{1}{t\left(a_{2}-a_{4}\right)}(d z-d t), \\
\omega^{4}=-t\left(a_{4} d x+a_{2} d y\right), \quad \omega^{3}=z\left(a_{3} d x+a_{1} d y\right)+\frac{1}{\left(a_{2}-a_{4}\right)}\left(a_{2} d z-a_{4} d t\right), \\
a_{1}+a_{3}=2 a_{2}, \quad a_{2}+a_{4}=2 a_{3} .
\end{gathered}
$$

ЗАмЕчАниЕ 7. Некоторые уравнения на тензоры кривизны пространства $M^{4}$ связаны с ОДУ. Например, из уравнения

$$
R_{i j ; k}+R_{j k ; i}+R_{k i ; j}=0
$$

следуют ограничения на коэффициенты $a_{i}(x, y)$ :

$$
\begin{gathered}
\alpha_{x}^{\prime \prime}+2 a_{3} \alpha^{\prime \prime}-2 a_{4} \alpha^{\prime}=0, \\
\alpha_{y}+2 a_{1} \alpha^{\prime}-2 a_{2} \alpha=0, \\
\alpha_{y}^{\prime \prime}+2 \alpha_{x}^{\prime}+4 a_{2} \alpha^{\prime \prime}-2 a_{4} \alpha-2 a_{3} \alpha^{\prime}=0, \\
\alpha_{x}+2 \alpha_{y}^{\prime}-4 a_{3} \alpha+2 a_{2} \alpha^{\prime}+2 a_{1} \alpha^{\prime \prime}=0 .
\end{gathered}
$$

Решениями этой системы будут дифференциальные уравнения второго порядка, связанные с пространством $M^{4}$, где на тензор Риччи наложено условие (5). Простейшие примеры таких уравнений имеют вид

$$
y^{\prime \prime}-\frac{3}{y} y^{\prime 2}+y^{3}=0, \quad y^{\prime \prime}-\frac{3}{y} y^{\prime 2}+y^{4}=0 .
$$




\section{4. ГЕОМЕТРИЯ ЭЙНШТЕЙНА-ВЕЙЛЯ В ТЕОРИИ ОДУ ВТОРОГО ПОРЯДКА}

Соотношения, возникаюшие между уравнениями вида (1) и уравнением $b^{\prime \prime}=$ $g\left(a, b, b^{\prime}\right)$, где функция $g\left(a, b, b^{\prime}\right)$ удовлетворяет ОДУ

$$
\begin{aligned}
g_{a a c c}+ & 2 c g_{a b c c}+2 g g_{a c c c}+c^{2} g_{b b c c}+2 c g g_{b c c c}+ \\
& +g^{2} g_{c c c c}+\left(g_{a}+c g_{b}\right) g_{c c c}-4 g_{a b c}-4 c g_{b b c}- \\
& -c g_{c} g_{b c c}-3 g g_{b c c}-g_{c} g_{a c c}+4 g_{c} g_{b c}-3 g_{b} g_{c c}+6 g_{b b}=0,
\end{aligned}
$$

были исследованы Картаном [13] с геометрической точки зрения. Он показал, что тройки Эйнштейна-Вейля параметризуют семейства кривых, описываемых решениями уравнения (5), которое оказывается дуальным уравнению (1). Некоторые частные решения уравнения (5) были получены в работе [2].

Рассмотрим теперь примеры пространств Эйнштейна-Вейля. Приведем вкратце основные сведения об этих пространствах (см. [14]).

Пространство Вейля - это гладкое многообразие с заданной конформной метрикой $g_{i j}(x)$, с симметрической связностью

$$
G_{i j}^{k}=\Gamma_{i j}^{k}-\frac{1}{2}\left(\omega_{i} \delta_{j}^{k}+\omega_{j} \delta_{i}^{k}-\omega_{l} g^{k l} g_{i j}\right)
$$

и с условием ковариантного дифференцирования $D_{i} g_{k j}=\omega_{i} g_{k j}$, где $\omega_{i}(x)$ - компоненты векторного поля.

Тензор кривизны, отвечаюший связности Вейля $G_{i j}^{k}$, имеет вид $W_{j k l}^{i}$, и тензор Риччи $W_{j i l}^{i}$ не обязан быть симметричным в обшем случае: $W_{j i l}^{i} \neq W_{l i j}^{i}$.

Пространство Вейля, удовлетворяюшее условию Эйнштейна

$$
\frac{1}{2}\left(W_{j l}+W_{l j}\right)=\lambda(x) g_{j l}(x)
$$

для некоторой функции $\lambda(x)$, собственно и называется пространством ЭйнштейнаВейля.

В трехмерном пространстве с метрикой $d s^{2}=d x^{2}+d y^{2}+d z^{2}$ компоненты вейлевской связности имеют вид

$$
2 G_{1}=\left|\begin{array}{ccc}
-\omega_{1} & -\omega_{2} & -\omega_{3} \\
\omega_{2} & -\omega_{1} & 0 \\
\omega_{3} & 0 & -\omega_{1}
\end{array}\right|, \quad 2 G_{2}=\left|\begin{array}{ccc}
-\omega_{2} & \omega_{1} & 0 \\
-\omega_{1} & -\omega_{2} & -\omega_{3} \\
0 & \omega_{3} & -\omega_{2}
\end{array}\right|, \quad 2 G_{3}=\left|\begin{array}{ccc}
-\omega_{3} & 0 & \omega_{1} \\
0 & -\omega_{3} & \omega_{2} \\
-\omega_{1} & -\omega_{2} & -\omega_{3}
\end{array}\right| .
$$

Из уравнений пространства Эйнштейна-Вейля

$$
W_{[i j]}=\frac{W_{i j}+W_{j i}}{2}=\lambda g_{i j}
$$

получается система уравнений

$$
\begin{gathered}
\omega_{3 x}+\omega_{1 z}+\omega_{1} \omega_{3}=0, \quad \omega_{3 y}+\omega_{2 z}+\omega_{2} \omega_{3}=0, \quad \omega_{2 x}+\omega_{1 y}+\omega_{1} \omega_{2}=0, \\
2 \omega_{1 x}+\omega_{2 y}+\omega_{3 z}-\frac{\omega_{2}^{2}+\omega_{3}^{2}}{2}=2 \lambda, \quad 2 \omega_{2 y}+\omega_{1 x}+\omega_{3 z}-\frac{\omega_{1}^{2}+\omega_{3}^{2}}{2}=2 \lambda, \\
2 \omega_{3 z}+\omega_{2 y}+\omega_{1 x}-\frac{\omega_{1}^{2}+\omega_{2}^{2}}{2}=2 \lambda .
\end{gathered}
$$


Первые три из этих уравнений приводят к уравнению Шази [15] $R^{\prime \prime \prime}+2 R R^{\prime \prime}-3 R^{\prime 2}=0$ на функцию

$$
R=R(x+y+z)=\omega_{1}+\omega_{2}+\omega_{3},
$$

где $\omega_{i}=\omega_{i}(x+y+z)$, и эти функции задают обобшение классического уравнения Шази.

Геометрия Эйнштейна-Вейля для метрики $g_{i j}=\operatorname{diag}\left(1,-e^{U},-e^{U}\right)$ и вектор $\omega_{i}=$ $\left(2 U_{z}, 0,0\right)$ определяются решениями уравнения [16]

$$
U_{x x}+U_{y y}=\left(e^{U}\right)_{z z}
$$

которое после подстановки $U=U(x+y=\tau, z)$ становится эквивалентным уравнению

$$
U_{\tau}=\left(e^{U / 2}\right)_{z}
$$

допускаюшему многозначные решения.

Рассматривая структуру Эйнштейна-Вейля для метрики $d s^{2}=d y^{2}-4 d x d z-4 u d t^{2}$, получим бездисперсионное уравнение КП [17] $\left(U_{t}-U U_{x}\right)_{x}=U_{y y}$.

Благодарности. Автор благодарит физический факультет университета Рим 1 и университет г. Лечче за гостеприимство. Работа частично поддержана фондом MURST (Италия), Фондом Карипло (Научньй центр Ландау-Вольта, Комо, Италия) и INTAS (грант № 99-01782).

\section{Список литературы}

[1] V. Dryuma. Buletinul AS RM (mathematica), Kishinev. 1999. V. 3. № 31. P. 95-102.

[2] V. Dryuma. Mathematical Researches (Kishinev). 1990. V. 112. P. 93-103.

[3] V.S. Dryuma. On initial values problem in theory of the second order ODE's. In: Proc. Workshop on Nonlinearity, Integrability, and All That: Twenty years after NEEDS'79. Gallipoli (Lecce), Italy, July 1-July 10, 1999,. Eds. M. Boiti, L. Martina, F. Pempinelli, B. Prinari, G. Soliani. Singapore: World Scientific, 2000. P. 109-116.

[4] В. С. Дрюма. О геометрии дифференциальных уравнений второго порядка. В сб.: Труды всесоюзной конференции "Нелинейные уравнения". Ред. К.В. Фролов. М.: Наука, 1991. C. $41-48$.

[5] В. С. Дрюма. ТМФ. 1994. Т. 99. С. 241-249.

[6] V.S. Dryuma. Geometrical properties of nonlinear dynamical systems. In: Proc. First Workshop on Nonlinear Physics. Le Sirenuse, Gallipoli (Lecce), Italy, June 29-July 7, 1995. Eds. E. Alfinito, M. Boiti, L. Martina, F. Pempinelli. Singapore: World Scientific, 1996. P. 83-93.

[7] R. Liouville. J. École Polytec. 1889. V. 59. P. 7-76.

[8] A. Tresse. Détermination des Invariants Ponctuels de l'Équation Differentielle Ordinaire de Second Ordre: $y^{\prime \prime}=w\left(x, y, y^{\prime}\right)$. Preisschriften der fürstlichen Jablonowski'schen Gesellschaft. V. 32. Leipzig: S. Hirzel, 1896.

[9] A. Tresse. Acta Math. 1894. V. 18. P. 1-88.

[10] E. Cartan. Bull. Soc. Math. France. 1924. V. 52. P. 205-241.

[11] E. M. Paterson, A. G. Walker. Quart. J. Math. Oxford. 1952. V. 3. P. 19-28.

[12] E. Wilczynski. Trans. Am. Math. Soc. 1908. V. 9. P. 103-128.

[13] E. Cartan. Ann. École Normale Sup. 1943. V. 14. P. 1-16.

[14] H. Pedersen, K. P. Tod. Adv. Math. 1993. V. 97. P. 71-109.

[15] J. Chazy. C. R. Acad. Sci. Paris. 1910. V. 150. P. 456-458.

[16] R. Ward. Class. Q. Grav. 1980. V. 7. P. L45-L48.

[17] M. Dunaisjski, L. Mason, P. Tod. Einstein-Weyl geometry, the dKP equation, and twistor theory. math.DG/0004031. 